\title{
Research Status, Key Technology and Its Application Prospect of Complex Products Assembly Quality Control and Prediction Based on Digital Twin
}

\section{Xuepeng Guo ( $\nabla$ guoxuepeng_2020@163.com ) \\ NUST: Nanjing University of Science and Technology \\ Linyan Liu \\ NUST: Nanjing University of Science and Technology \\ Huifeng Wang \\ NUST: Nanjing University of Science and Technology \\ Tangxiao Yuan \\ NUST: Nanjing University of Science and Technology}

\section{Research Article}

Keywords: Digital Twin, Quality Control and Prediction, Modeling and Simulation, Data Analysis and Decision Makin

Posted Date: May 7th, 2021

DOl: https://doi.org/10.21203/rs.3.rs-438909/v1

License: (c) (i) This work is licensed under a Creative Commons Attribution 4.0 International License. Read Full License 


\section{Abstract}

In order to solve the problem of unmeasurable assembly performance of complex product, the digital twin-driven assembly quality control and prediction of complex product is studied by means of cyberphysical fusion in the assembly workshop. The connotation of digital twin intelligent assembly is introduced, the current research status of complex product assembly quality is compared and analyzed, and three main key technologies for the assembly quality control of complex product are proposed: (1) multidimensional, multi-scale, multidisciplinary modeling and simulating of digital twin-driven assembly; (2) multi-source heterogeneous data collection, sensing and fusion for assembly processes; (3) datadriven decision making, feedback and optimization technology. Finally, the application of digital twin technology in the field of assembly quality control of complex product is prospected.

\section{Introduction}

Complex products are a class of products with complex technology, complex component composition, complex manufacturing processes, complex relationship between components and products such as satellites, aircraft, weapon systems, antenna servo systems. Which are characterized by high complexity, high precision, high reliability, strong coupling, and high value[1]. Assembly refers to assemble various parts in accordance with the established technical requirements, and make it to be a qualified product after debugging and inspecting[2]. Assembly occupies $20 \%-70 \%$ of the workload in the actual production process, this is a crucial part of the product development process. Assembly accuracy, stability and efficiency and other quality issues are important indicators to measure whether the equipment is qualified in the assembly process of complex product, which directly affect the whole manufacturing and service process of complex product. Therefore, the optimization and prediction of assembly quality will have important engineering significance to improve the overall performance of complex product[3].

With research \& development demand of intelligence, integration, and lightweight, the new generation of high precision and complex products are developing towards multi-functional integration and array, and the traditional product assembly has the following problems: (1) poor assembly reliability. There are many different connection methods of components in mechanical assembly such as screw fastening, tapered plus nut fastening, key connection, spline connection, coupling connection, interference fit connection, riveting, welding. this complicated connection methods will inevitably lead to irregular deformation, traditional simulation means cannot predict real-timely the stress changes in the assembly process, it will produce irreversible damage and affect seriously the reliability of connection points once appearing plastic deformation; (2) poor assembly consistency under non-visible conditions. Cumulative errors and dynamic assembly deformation can cause inconsistent connector gap due to the blind-install of numerous structural parts and electronic components in the new generation of high precision complex product, and there are no physical methods to measure the blind connection gap for online adjusting, this will affect seriously the assembly accuracy and performance; (3) poor reliability of mechanicalelectromagnetic coupling under-sampled conditions. Mechanical assembly accuracy has a complex correlation to electromagnetic performance due to the presence of many electronic components in the 
assembly process of high precision products, and the traditional assembly method cannot dynamically predict the impact of assembly parameters on electromagnetic performance, one method is to assemble and adjust repeatedly by electrifying in darkroom, which is still difficult to achieve the best performance index.

Traditional product assembly quality control methods are based on premise assumptions and ignore correlated influencing factors and cannot interact with the real world, which is difficult to achieve high confidence quality control and high accuracy performance prediction. It provides an effective solution to these bottlenecks when the emergence of digital twin technology. The digital twin-driven assembly process is an internet of things that integrates all equipment, this assembly process will achieve a deep integration of the physical worlds and information worlds, realize the accurate control of parts, equipment and assembly process through intelligent software service platforms and tools, and realize the selforganization, self-adaptation and dynamic response of the product assembly system [4]. Therefore, it is an urgent need for digital twin technology to achieve many key difficulties such as online real-time simulation, blind area visibility, performance prediction, feedback control in the assembly process of complex product, and to solve the problem of unpredictable assembly performance.

\section{The Connotation Of Intelligent Assembly Based On Digital Twin 2.1 The concept of digital twin}

The conceptual model of the digital twin was first introduced in 2003 by Professor Grieves M.W. in a product lifecycle management course at the University of Michigan, and was originally called the "Mirrored Spaced Model"[5]. In 2010, NASA first introduced the digital twin concept to the space technology roadmap, with the goal of using digital twin technology for diagnostic and predictive functions of flight systems[6]. There is no single definition about digital twin. Its main idea is to digitally create virtual models of physical entities, simulate the behavior of physical entities in the real environment by data, and extend new capabilities to physical entities by means of virtual-real interaction feedback, data fusion analysis, and iterative optimization of decisions. As a technology that makes full use of models, data, intelligence and integrates multiple disciplines, digital twin is oriented to the whole product lifecycle process, playing the role of a bridge and link between the physical world and the information world to provide more real-time, efficient and intelligent services[7].

\subsection{Overview of digital twin driven intelligent assembly}

Intelligent assembly based on digital twin will solve assembly quality problems in the assembly process through interactive feedback, data fusion and analysis, and iterative optimization of decisions between the virtual assembly workshop and the real physical assembly workshop[8]. The digital twin-driven intelligent assembly consists of physical assembly workshop, cyber assembly workshop, and workshop digital twin data, as shown in Fig. 1. 
The physical assembly workshop is a collection of various physical entities such as devices, equipment, materials, and personnel in the assembly process, this workshop is mainly consisting of assembly production lines, assembly material distribution, assembly processes, assembly execution, and other systems. The physical assembly workshop accomplishes specific tasks through the collaboration among systems; in addition, the environmental data and operation status of assembly process can be monitored in real time by various sensors deployed on the physical entities, which further sense and control the workshop production process.

\section{(2)Cyber assembly workshop}

The cyber assembly workshop is a high-fidelity mirror of various production elements such as personnel, machines, materials, and environment of the physical assembly workshop, and is a full-factor mapping from physical assembly workshop to cyber assembly workshop. On the one hand, the assembly state and process of complex product in the physical assembly workshop can be mapped dynamically, real-time and accurately to the cyber assembly workshop, which would realize dynamic perception, selfdetermination and accurate control of the assembly process; on the other hand, in order to support the full element mapping from cyber assembly workshop to physical assembly workshop, the definition and planning information of the cyber assembly workshop should be accurately synchronized to the corresponding entity in the physical assembly workshop.

\section{(3)Workshop digital twin data}

The workshop digital twin data consists of actual measurement data from the physical assembly workshop, soft sensing data such as simulation, calculation and mechanism from cyber assembly workshop, and fusion data from virtual-real interaction. During the operation of the digital twin system, data from physical assembly workshop is collected in real time and synchronized to the cyber assembly workshop, then digital twin model will be updated by fusing soft sensing data and the design model and form the basis for simulation prediction model. This prediction model will drive the assembly system to complete the assembly process after making process decisions based on the simulation prediction results. In addition, the soft sensing data will synchronize again to the cyber assembly workshop for monitoring and entering next simulation prediction, and finally forming a continuous interactive and synchronized evolution of workshop data from product assembly process.

\section{Research Status Of Complex Product Assembly Quality}

Complex product has high precision and high density, cross-scale integration, strong mechanicalelectromagnetic coupling and other characteristics, its assembly quality directly affects the core performance of the equipment. For complex product, the assembly process will inevitably introduce deformation, errors, and other problems, which will lead to change relative to the expected performance of mechanical properties and electromagnetic properties in actual assembly of complex product. Therefore, the consistency, stability and controllability become the key to determine the quality of complex product assembly. 


\subsection{Product intelligent assembly}

The assembly deformation, blind insertion error of parts, uncontrollable assembly error, invisible assembly state and other problems will lead to product assembly accuracy, design requirements cannot meet the requirements in the actual assembly process of complex product. The traditional assembly method cannot predict dynamically the assembly error and reliability, and can only improve the reliability of the assembly by eliminating errors manually, which is inefficient and laborious. For high-precision integrated products, the assembly process cycle is longer and the uncertainty of assembly process is greater than others, so the traditional assembly method is difficult to achieve the best performance index.

\subsubsection{Technology of virtual assembly}

Virtual assembly is a technology that achieve interactive analysis, simulation of assembly process, simulation of assembly results after constructing virtual models in virtual environment and realizing human-computer interaction in assembly process by using virtual reality technology, computer graphics, artificial intelligence technology[9]. The core of virtual assembly technology is modeling and simulation, this technology can realize process of physical assembly by modeling and simulation and handle various problems and phenomena during the assembly process by simulating current assembly scene. in the 1990s, the National Institute of Standards and Technology (NIST) and Washington State University developed a virtual assembly system: Virtual assembly design environment (VADE), which can provide the most ideal sequence of parts assembly for assembly personnel, as well as generate assembly/disassembly paths. With the deeper research of virtual assembly technology, the virtual assembly process planning system[10,11], the assembly sequence planning in virtual environment[12$15]$, the modeling method of assembly process $[16,17]$, the assembly simulation[18-20]and other related design and manufacturing activities have improved the efficiency and quality of product assembly, which greatly compensate the disadvantages and shortcomings of traditional assembly methods.

\subsubsection{Assembly technology based on digital twin}

In recent years, with the development and application of digital twin technology, there are related scholars have done some research in the assembly problem of complex product. Bilberg[21] proposed a humanrobot collaborative assembly control method based on digital twin in order to reduce the risk factor of personnel and frequent configuration requirements in the assembly process, it could solve the workload balance between human and robot of various product assembly process and dynamically balance the workload during operation by constructing digital twin human-robot collaboration model; Aderiani[22, 23] proposed an adjustment method of assembly fixture positioner based on digital twin to improve the assembly geometry quality, the method could achieve the effect of adjusting the part compensation by adjusting the positioner through the simulation results which from constructed digital twin simulation model by real-time scanning data; Wang[24] proposed a spacecraft manufacturing/assembly system based on digital twin in his application framework of spacecraft system, this system could achieve deep integration of the information world and the physical world by building a physical workshop and high- 
fidelity digital workshop, it also could realize dynamic sensing, real-time analysis, self-determination, precise control and visualization in manufacturing/assembly process; In order to realize the informationphysical integration of complex product design and manufacturing, Li[25] proposed a integration framework for complex product design and manufacturing based on digital twin, and explored the key implementation technologies such as requirements analysis, conceptual design, personalized configuration design, virtual prototyping, multidisciplinary fusion design and data management during the development process; In order to solve complex assembly process, high requirements, variable technical states and other key difficulties in aero-engine assembly, Sun[26] proposed a digital twin-driven aero-engine assembly technology from the key technologies such as assembly process control, parts selection, assembly operation guidance, assembly gap control, assembly technology state control and assembly test data correlation analysis, which effectively improved the aero-engine assembly success rate and quality performance; Zhang[27] proposed to construct a digital twin of spacecraft in-orbit assembly to express the process, state, and behavior of spacecraft assembly due to the lack of simulation, monitoring, diagnosis, and prediction processes in spacecraft assembly.

The emergence and rapid development of digital twin technology has gradually come into people's view. As a fundamental element in intelligent manufacturing, digital twin provides a new direction for complex product assembly. Through the interactive integration of physical assembly workshop, digital assembly workshop and workshop digital twin data, it can realize real-time control of the assembly process and improve assembly intelligence, initiative and prediction, and assembly quality and level would be great improved.

\subsection{Assembly performance analysis}

The assembly performance analysis is an important issue in product assembly. A series of assembly mechanical performance problems such as deformation, stress, and assembly error transmission during the assembly process can greatly reduce the assembly accuracy and assembly success rate. In view of the above problems, some related scholars have made relevant studies and achieved many research results and mainly involving assembly error analysis, assembly stress analysis, assembly deformation analysis and assembly electromagnetic performance analysis. The traditional analysis methods focus on simulation of assembly deviation models based on an ideal surface in assembly error analysis [28-30], which ignores the shape defects of components and assembly systems. With the development of computer aided design and computer aided manufacturing technology, the assembly tolerance analysis method based on skin model shape has been widely used in the field of product assembly tolerance analysis[31-34], and has achieved satisfactory results. In recent years, with the rapid development of digital twin technology, many scholars and researchers have proposed a geometric deviation management method based on digital twin in the product assembly process[35, 36], this method of simulating the whole assembly process has achieve accurate analysis and prediction of assembly errors through the interactive fusion of real physical model and high-fidelity virtual model, and provided a theoretical basis for improving the stability and quality of product assembly. In assembly stress analysis, the generated stress by the assembly process not only seriously affects the structural stability of the 
mechanical system, but also plays a crucial role in the assembly accuracy and reliability of the mechanical system. McCune[37] has analyzed the influence of friction stir welding(FSW) process on residual stress and distortion predictions for future airframe assembly simulations; Yao[38] proposed a high-precision gyroscope assembly stress control method; Paulo[39] proposed a novel shell-based finite element model to accurately simulate the transient thermal fields and stress-strain distributions resulting from friction stir welding processes; $\mathrm{Li}[40]$ analyzed the spot welding stress of auto-body assembly. In assembly deformation analysis, the positioning, clamping methods and the assembly connection methods (such as riveting and welding) of the assembly process will result in different deviation variations and part deformation, and lead to poor assembly reliability and reduced assembly quality and performance. Wang[41-43] conducted a deformation analysis of the array antenna reflector panel assembly and proposed an optimization method for the array antenna panel assembly; Pan[44] proposed a numerical simulation analysis for deformation deviation for an antenna thin-wall parts considering riveting assembly with finite element method; Qing[45] created a Positioning variation modeling for aircraft panels assembly based on elastic deformation theory. In assembly electromagnetic performance analysis, the electromagnetic performance of products is easily affected by the assembly process, and the inconsistency of assembly accuracy often leads to complex product electromagnetic performance cannot meet the requirements. Mahajan[46] proposed a performance prediction method of electromagnetic band gap structure for microstrip antenna using FDTD-PBC unit cell analysis and Taguchi's multi-objective optimization; Nair[47] analyzed the electromagnetic design and performance analysis of airborne radomes, and described the trends and prospects; Zhou[48-51] has done a lot of research on the relationship between mechanical structure and electrical properties, and has achieved more research results; In addition, Xu[52] analyzed the effect of electrical performance of metal space frame radome with structural deformation, and proposed a new method to describe the radome deformation.

There is a common problem in the product manufacturing process: the accuracy and reliability of the product cannot meet the overall design requirements due to the existence of deformation of parts, stress, error transmission and mechanical-electromagnetic coupling after finishing the assembly in the case of the parts design and parts processing meet technological requirements. Therefore, how to predict the assembly accuracy and performance to achieve real-time control of the quality problems before product assembly process will have important research significance for the manufacture of high-quality products.

\subsection{Assembly quality control}

Electronic equipment assembly is often assembled by many processes to assemble the parts together, in this process, product quality and each process is closely linked together, the quality data of the assembly process is an important basis for evaluating the quality of complex product assembly. It will generate very important research significance to improve assembly success rate of complex product by controlling and analyzing assembly quality of complex product. Due to the characteristics of intelligence, integration, and lightweight, the traditional assembly mode cannot satisfy the high-quality requirements of complex 
product assembly, and the product assembly is gradually transformed to virtualized and intelligent assembly mode.

Cai[53] proposed a quality management and analysis method for aircraft assembly based on digital twin with multi-source heterogeneous and inconsistent formats of quality data; Wagner[54] introduced a method to integrate product functional knowledge into production control based on digital twin; Ma[55] established a digital twin system for interactive mapping between the physical world and digital world in the assembly process, this system has solved the deficiencies of insufficient quality analysis, control and decision-making capabilities in the assembly process of complex products through integration, analysis and collaborative optimization from multi-source heterogeneous data; Zhuang[56-58] proposed a intelligent control method based on digital twin due to the high dynamics, uncertainty, and frequent rework in complex product assembly workshop; Weng[59] proposed an aviation products assembly process quality data collection and management method for the problem of difficult quality data management and poor traceability; Meng[60] proposed a digital twin-driven robot automatic assembly control method; $\mathrm{Wu}[61]$ introduced a quality control method for complex products assembly process based on digital twin. The above studies provide a certain theoretical basis for the development of complex product assembly quality control based on digital twin. However, due to the strong mechanicalelectromagnetic coupling of complex product, it has the influence such as mechanical, electromagnetic and mechanical-electromagnetic coupling properties in assembly process, which need to be combined with simulation data to conduct the complex product assembly quality control research from different performance perspectives; in addition, the above methods lack relevant online prediction research of assembly performance, which cannot achieve a knowable, credible and controllable assembly process.

In the assembly process of complex product, it has a very important significance for ensuring the assembly quality by making objective, accurate decisions, and active prevention before assembly. it provides a new way to improve the production efficiency and optimize the assembly quality of complex

product by assembly quality control based on digital twin. this method will promote the development and application of theories, methods and tools of complex product assembly simulation and online performance prediction through the study of real-time control and online prediction, and will form a new mechanism with knowable assembly state, credible assembly performance and easy control of the assembly process, achieve accurate prediction and effective mapping of complex product assembly quality. this will have important engineering application value and research significance.

\section{Digital Twin-driven Analysis Of Key Technologies For Complex Product Assembly Quality Control And Prediction}

The key to complex product assembly based on digital twin is to construct a cyber-physical fusion system, whose assembly process is a physical and virtual assembly co-evolutionary process. In order to achieve "controllable and measurable" in assembly quality, it is first necessary to build a multidimensional, multi-scale, and multidisciplinary high-fidelity model of complex product, and use the digital twin high-fidelity model for assembly simulation; Secondly, it is necessary to realize the real-time 
collection, sensing and fusion of multi-source heterogeneous data in the assembly process; Finally it is necessary to combine physical and virtual data fusion technology to realize dynamic data analysis, decision-making and optimization, and build an online accurate prediction model of assembly performance, realize intelligent simulation and online accurate prediction of assembly performance based on digital twin.

\subsection{Multidimensional, multi-scale, multidisciplinary modeling and simulating of digital twin-driven assembly}

The core of the digital twin is the mapping of physical entities in virtual space. The fidelity of the virtual model determines the success of the application of digital twin assembly technology[62]. In the digital twin-driven assembly process, the virtual assembly workshop guides the physical assembly workshop to complete assembly tasks by simulating the assembly behavior of the physical assembly workshop.

Therefore, establishing a high-fidelity virtual model of the virtual assembly workshop based on geometry, physics, behavior, and rules, and satisfying the simultaneous evolution of the virtual model and physical perception data are the core of realizing the quality control and prediction of complex product assembly based on the digital twin.

Unlike traditional complex products, the assembly problems of complex product are not only reflected in pure structural performance such as structural fatigue, strength, and stiffness, but also in electrical performance such as electromagnetic and signal processing, and electromechanical coupling. Therefore, in complex product assembly quality control and prediction, it is necessary to establish a multi-scale (system, sub-assembly, parts, etc.), multidimensional (function, performance, behavior, etc.), multidisciplinary (mechanical, electromagnetic, etc.) virtual model. At the same time, the virtual model is required to have multi-scale, multidimensional, multidisciplinary simulation, analysis, and prediction capabilities. The simulation of the assembly process in the virtual environment needs to ensure mechanical performance, and needs to ensure or even improve electrical performance to realize the mechatronics of complex product assembly[63].

Each physical performance of the complex product assembly process corresponds to a specific model, such as assembly error model, assembly deformation model, electromagnetic performance model and electromechanical coupling model. It is the key to the quality prediction and control of complex product assembly that how to associate these models with different physical characteristics. The multi-physics integrated modeling can not only improve the accuracy of virtual simulation, and the simulation results can also reflect the state and behavior of the physical assembly more realistically, making it possible to replace the physical prototype with digital twin-driven assembly[64]. Therefore, multi-scale, multidimensional, multidisciplinary modeling and simulation will be an important technical mean to improve the fidelity of virtual models and give full play to the role of digital twin.

\subsection{Multi-source heterogeneous data collection, sensing and fusion for assembly processes}


The collection and transmission of high-fidelity sensor data is the basis for establishing a digital twin model of complex product. The complex product assembly process generates a large amount of assembly data, which include soft sensor data such as simulation data, calculation data and mechanism data of the digital twin workshop, and actual measurement data of the physical assembly workshop. With the help of OPC communication protocol, RFID, ultrawide band (UWB) and other technologies[65, 66], the assembly process quality data will be collected and stored in the virtual assembly workshop and the physical assembly workshop, and through the analysis of each assembly quality data, the reducedorder characterization model between complex product parts will be constructed, and then the coupled reduced-order accuracy correction will be completed based on deep learning algorithms to establish the agent model in the assembly process of complex product, which is an important way to realize online quality control and prediction of complex product assembly process based on digital twin[67]. In addition, in the process of complex product assembly, the data collected by various sensor devices has the characteristics of grammatical, semantic, and structural heterogeneity, which is a huge challenge for multi-source heterogeneous data collection and fusion.

The assembly process quality data collection methods mainly include software collection, hardware collection and manual collection. Software collection is the indirect collection from other systems through software integration interfaces and database sharing; hardware collection is the direct collection of assembly process quality data through measurement, sensors, and other instruments; manual collection refers to part of the assembly quality data needs to be manually recorded and stored in the actual assembly process, and manual collection mainly relies on manual measurement and input. The assembly process quality data collection process is shown in Fig. 2.

In the process of complex product assembly, the types, attributes, and content of collected data are very different. So, it is necessary to study the standard data communication and conversion interface, realize the unified conversion and unified encapsulation of different types of data, and convert the multi-source heterogeneous data into definite data that computer-recognizable and required for digital twin simulation analysis. On this basis, it is also necessary to plan, clean, excavate, cluster, and package the real-time data, simulation data and mechanism data covering the assembly process of complex product to achieve data traceability and operability. Through the operation of classification, association, and combination of multi-source heterogeneous data, it is helpful to accurately depicts and reflects the state of dynamic evolution process, evolution law, statistical features, and other development process, and improve the accuracy of state and feature estimation, and provide real-time basic data support for the construction of digital twin model.

\subsection{Data-driven decision making, feedback and optimization technology}

In the assembly process of complex product, the assembly progress, assembly accuracy and reliability are monitored, adjusted, and optimized based on theoretical values and mechanistic models through the interactive fusion, analysis and decision making of multi-source heterogeneous data. The digital twin- 
driven feedback technology assists assemblers to strengthen the closed-loop feedback control of the assembly process through the comparative analysis of physical data and design data, which will realize real-time monitoring of the assembly process and achieve quality control and online accurate prediction of the assembly performance.

During the assembly stage, various sensors are used to track and monitor the assembly status such as mechanical properties, electromagnetic properties, environmental conditions, and operating parameters. These monitoring data and historical data can predict analyze the assembly performance and other indicators. Based on the digital twin data that fuse by physical measurement data and virtual simulation data, the latest measurement data, schedule data, performance data, and measurement values of assembly process state parameters are mapped to the digital twin model, and this will realize a closely connection with the real world and the information world based on the digital twin assembly model. Similarly, the big data analysis methods such as Markov method[61], D-S evidence theory[68-70], Taguchi method[71, 72], support vector machine[73] will also be used in the stage of data analysis and decision making to achieve assembly quality control and prediction, which helps reduce assembly errors and improve assembly efficiency.

\section{Application Prospect Of Digital Twin Technology In Complex Product Assembly Quality Control}

It will realize the control and online prediction of complex product assembly quality by using the method of "virtual and real symbiosis, virtual control of real" when introducing digital twin technology into the quality control of complex product assembly. Digital twin technology can comprehensively solve the problems of unpredictable assembly performance, uncontrollable assembly process, and invisible assembly status, and realize high-performance, high-consistency, and reliable assembly in the process of complex product assembly quality control. Around the digital twin-driven complex product assembly quality control research, it is urgent to make a breakthrough in the following difficult issues:

(1) High-fidelity modeling of complex product assembly quality control system. Live and constructive symbiotic is the main feature of digital twin-driven complex product assembly quality control, it can provide a theoretical basis for assembly quality control data flow management by constructing a highfidelity model of the complex product assembly process.

(2) Multiple integration of digital twin data in the assembly process. Digital twin data is the bridge connecting the physical assembly workshop and the cyber assembly workshop, and is the key foundation for realizing the quality control of complex product assembly. The multiple interaction and fusion of digital twin data promotes the operation of physical assembly workshop and cyber assembly workshop, and is the core driver to realize the quality control of complex product assembly.

(3) Information-physical fusion of assembly quality control system. Information-physical fusion is a prerequisite for the development of complex product assembly quality control. The simulation of complex 
product assembly process, the construction of assembly quality control model, and the performance evaluation and prediction of assembly process all need to study the method of information-physical fusion between physical assembly workshop and cyber assembly workshop driven by live and constructive symbiotic.

\section{Conclusion}

Under the concept of digital twin, this paper has explored the current status of research, key technologies and application prospects of complex product assembly quality control based on digital twin. The current research status of complex product assembly quality control has been explained and summarized from the aspect of demand analysis; the key technologies of digital twin-driven complex product assembly quality control have been analyzed from the aspects of virtual simulation, data modeling, and online prediction; finally, the application prospects of digital twin technology in complex product assembly quality control have been explored. At present, the research on digital twin-driven assembly quality control technology for complex product is in the development stage, and the key technologies and application prospects of the research need to be further studied and explored. It is hoped that this paper can provide some theoretical and technical references for the future application of digital twin technology.

\section{Declarations}

a. Funding: This work was supported by the National Natural Science Foundation of China under Grant 51705256.

b. Conflicts of interest/Competing interests: The authors declare that there is no conflict of interests regarding the publication of this article.

c. Availability of data and material: All data generated or analyzed during this study are included in this published article.

d. Code availability: Not applicable.

e. Ethics approval: Not applicable.

f. Consent to participate: Not applicable.

g. Consent for publication: Not applicable.

\section{References}

1. Hu X, Liu JH, Zhuang CB, Wu Y, Zhang L, Xiong H (2021) Digital twin-based management method and application for the complex products assembly process. Comput Integr Manuf Syst 27(2):642653

2. Liu JH, Sun QC, Cheng H, Liu XK, Ding XY, Liu SL, Xiong H (2018) The State-of-the-art, Connotation and Developing Trends of the Products Assembly Technology. Journal of Mechanical Engineering 54(11):2-28 
3. Wang XQ, Liu MZ, Ling L, Liu CH (2015) Research on assembly quality adaptive control system for complex mechanical products assembly process under uncertainty. Comput Ind 74:43-57. http://dx.doi.org/10.1016/j.compind.2015.09.001

4. Tao F, Liu WR, Liu JH, Liu XJ, Liu Q, Qu T, Hu TL, Zhang ZN et al (2018) Digital twin and its potential application exploration. Comput Integr Manuf Syst 24(1):1-18

5. Grieves MW (2005) Product lifecycle management: the new paradigm for enterprises. International Journal of Product Development 2(1-2):71-84

6. Yang LY, Chen SY, Wang X, Zhang J, Wang CH (2019) Digital twins and parallel systems: state of the art, comparisons and prospect. Acta Automatica Sinica 45(11):2001-2031

7. Cimino C, Negri E, Fumagalli L (2019) Review of digital twin applications in manufacturing. Comput Ind 113:103130. https://doi.org/10.1016/j.compind.2019.103130

8. Qiu C, Zhou SE, Liu ZY, Gao Q, Tan JR (2019) Digital assembly technology based on augmented reality and digital twins: a review. Virtual Reality Intelligent Hardware 1(6):597-610

9. Ning RX, Zheng Y (2005) Analysis on recent Study progresses and trends of virtual assembly technology. China Mechanical Engineering 16(15):1398-1404

10. Wang CE, Yu, Hong Y, Yu JP, Zhang WL, Yuan H (2011) Assembly planning system for complex product. Comput Integr Manuf Syst 17(5):952-960

11. Dong L, Yu JF, Li Y, Wang YP, Zhang J (2012) Integrated model for aircraft assembly process planning and simulation under 3D environment. Comput Integr Manuf Syst 18(6):1158-1167

12. Li PY, Cui J, Gao F, Wang CF, Mao YS, Liao GH (2015) Research on the Assembly Sequence of a Ship Block Based on the Disassembly Interference Matrix. Journal of Ship Production Design 31(4):230240

13. Liu YJ, Gu TL, Xu ZB, Chang L (2013) Parallel assembly sequence planning based on improved genetic programming. Comput Integr Manuf Syst 19(6):1238-1248

14. Wang S, Sun ZZ, Guo JW, Zhang ZC (2014) Assembly sequence planning based on shuffled frog leaping algorithm. Comput Integr Manuf Syst 20(12):2991-2999

15. Xia PJ (2016) A hybrid approach for haptics-based intelligent assembly planning. Advances in Mechanical Engineering 8(10):1-15

16. Song C, Liu HY (2016) Multi-agent based context-aware assembly model. Comput Integr Manuf Syst 22(5):1205-1210

17. Bao JS, Li ZQ, Xiang Q, Wu DL, Zheng XH (2018) The modeling, evolutionary and application of quasi-physical virtual assembly. Journal of Mechanical Engineering 54(11):61-69

18. Zhang J, Wang P, Zuo M, Li Y, Xu ZJ (2018) Automatic assembly simulation of product in virtual environment based on interaction feature pair. J Intell Manuf 29(6):1235-1256

19. Zhang QS, Jin X, Zhang ZG, Zhang ZG, Shang K (2018) Assembly Method Based on Constrained Surface Registration. Journal of Mechanical Engineering 54(11):70-76 
20. Yang XD, Liu JH, ZhaoYF, Ma JT, Lv NJ (2019) Rigid and flexible hybrid assembly process simulation technology for complex mechatronic products. Comput Integr Manuf Syst 25(2):340-349

21. Bilberg A, Malik AA (2019) Digital twin driven human-robot collaborative assembly. Cirp AnnalsManufacturing Technology 68(1):499-502

22. Aderiani AR, Warmefjord K, Soderberg R, Lindkvist L (2019) Developing a selective assembly technique for sheet metal assemblies. Int J Prod Res 57(22):7174-7188

23. Aderiani AR, Warmejord $K$, Soderberg R, Lindkvist $L$ (2019) Individualizing locator adjustments of assembly fixtures using a digital twin. J Comput Inf Sci Eng. https://doi.org/10.1115/1.4043529

24. Wang JJ, Xiang YQ, He ZW (2019) Models and implementation of digital twin based spacecraft system engineering. Comput Integr Manuf Syst 25(6):1348-1360

25. Li H, Tao F, Wang HQ, Song WY, Zhang ZF, Fan BB, Wu CL, Li YP, Li LL, Wen XY, Zhang XS, Luo GF (2019) Integration framework and key technologies of complex product design-manufacturing based on digital twin. Comput Integr Manuf Syst 25(6):1320-1336

26. Sun HB, Yan JX, Wei XH, Chang ZY (2020) Digital Twin-driven aero-engine assembly technology. China Mechanical Engineering 31(7):833-841

27. Zhang YL, Zhang JP, Wang XD, Chen XB, Liu JH (2018) Digital twin technology for spacecraft onorbit assembly. Navigation Control 17(03):75-82

28. Cao J, Gao ZY, Gao JM, Xie JT (2020) Precise selective assembly method for complex mechanical products based on manufacturing tolerance. Comput Integr Manuf Syst 26(7):1729-1736

29. Guo FY, Liu JH, Wang ZQ, Zou F, Zhao XD (2020) Positioning error guarantee method with two-stage compensation strategy for aircraft flexible assembly tooling. J Manuf Syst 55:285-301. https://doi.org/10.1016/j.jmsy.2020.03.008

30. Xi X, Xiao DB, Li W, Lu K, Shi Y, Yuan C, Wu XZ (2020) Analysis of assembly errors and design of precise micro-regulator for micro-hemispherical resonator gyroscope (mHRG). Journal of Mechanical Engineering 56(13):77-83

31. Schleich B, Anwer N, Mathieu L, Wartzack S (2014) Skin Model Shapes: A new paradigm shift for geometric variations modelling in mechanical engineering. Comput Aided Des 50:1-15. http://dx.doi.org/10.1016/j.cad.2014.01.001

32. Schleich B, Anwer N, Mathieu L, Wartzack S (2015) Contact and mobility simulation for mechanical assemblies based on skin model shapes. J Comput Inf Sci Eng. http://dx.doi.org/10.1115/1.4029051

33. Yan X, Ballu A (2016) Toward an automatic generation of part models with form error. Procedia CIRP 43:23-28. https://doi.org/10.1016/j.procir.2016.02.109

34. Yan X, Ballu A (2018) Tolerance analysis using skin model shapes and linear complementarity conditions. J Manuf Syst 48:140-156. https://doi.org/10.1016/j.jmsy.2018.07.005

35. Grégorio JL, Lartigue C, Thiébaut F, Lebrun R (2020) A digital twin-based approach for the management of geometrical deviations during assembly processes. J Manuf Syst. 
https://doi.org/10.1016/j.jmsy.2020.04.020

36. Liu ZY, Zhou SE, Qiu C, Tan JR (2019) Assembly variation analysis of complicated products based on rigid-flexible hybrid vector loop. Proceedings of the Institution of Mechanical Engineers Part B: Journal of Engineering Manufacture 233(10): 2099-2114

37. McCune RW, Murphy A, Price M, Butterfield J (2012) The influence of friction stir welding process idealization on residual stress and distortion predictions for future airframe assembly simulations. $J$ Manuf Sci Eng. https://doi.org/10.1115/1.4006554

38. Yao ZX, Hu JZ, Zhang ZJ (2018) Assembly technology of structure stability control for high precision gyroscope. Journal of Mechanical Engineering 54(9):145-152

39. Paulo RMF, Carlone P, Paradiso V, Valente RAF, Teixeira DF (2017) Prediction of friction stir welding effects on AA2024-T3 plates and stiffened panels using a shell-based finite element model. ThinWalled Structures 120:297-306. http://dx.doi.org/10.1016/j.tws.2017.09.009

40. Li SL, Wei ZY, Lu BH (2014) Stress analysis of auto-body assembly based on ANSYS. Transactions of the China Welding Institution 35(11):55-58

41. Wang D, Duan XQ, Shao XD, Gao F (2020) Technology of assembly sequence planning for reflector. Comput Integr Manuf Syst 26(6):1679-1690

42. Wang D, Shao XD, Liu HL, Ge XB (2020) Assembly sequence planning for panels of reflector antenna based on hybrid algorithm. Comput Integr Manuf Syst 23(6):1243-1252

43. Wang D, Shao XD, Liu SM (2016) Simulation technologies of large reflector antenna assembly deformation. Comput Integr Manuf Syst 22(11):2542-2550

44. Pan MH, Tang WC, Xing Y, Ni J (2018) Numerical simulation analysis for deformation deviation and experimental verification for an antenna thin-wall parts considering riveting assembly with finite element method. Journal of Central South University 25(1):60-77

45. Wang Q, Hou RL, Li JX, Ke YL, Maropoulos PG, Zhang XZ (2018) Positioning variation modeling for aircraft panels assembly based on elastic deformation theory. Proceedings of the Institution of Mechanical Engineers Part B:Journal of Engineering Manufacture 232(14): 2592-2604

46. Mahajan RC, Vyas V, Sutaone MS (2020) Performance prediction of electromagnetic band gap structure for microstrip antenna using FDTD-PBC unit cell analysis and Taguchi's multi-objective optimization method. Microelectron Eng. https://doi.org/10.1016/j.mee.2019.111156

47. Nair RU, Jha RM (2014) Electromagnetic design and performance analysis of airborne radomes: trends and perspectives. IEEE Antennas Propag Mag 56(4):276-298

48. Wang CS, Wang Y, Wang W, Zhou JZ, Wang M, Wang ZH (2017) Electromechanical coupling based influence of structural error on radiation and scattering performance of array antennas. Electron Lett 53(14):904-905

49. Zhou JZ, Song LW, Du LG, Guo DL (2016) Influence of dynamic load on the mechanical and electrical performance of structurally integrated antenna. Journal of Mechanical Engineering 52(9):105-115 
50. Zhou JZ, Song LW, Huang J, Wang CS (2015) Performance of structurally integrated antennas subjected to dynamical loads. Int J Appl Electromagnet Mech 48(4):409-422

51. Zhou JZ, Duan BY, Huang J, Li HP (2009) Prediction of plane slotted-array antenna electrical performance affected by manufacturing precision. Journal of University of Electronic Science Technology of China 38(6):1047-1051

52. Xu WY, Li P, Qiu YY, Lin ZC (2016) Electrical performance analysis of metal space frame radome with structural deformation. Journal of Mechanical Engineering 52(1):57-63

53. Cai HX, Zhang W, Zhu Z (219) Quality management and analysis of aircraft final assembly based on digital twin (2019) 11th International Conference on Intelligent Human-Machine Systems and Cybernetics. https://doi.org/10.1109/IHMSC.2019.00054

54. Wagner R, Haefner B, Biehler M, Lanza G (2020) Digital DNA in quality control cycles of highprecision products. CIRP Ann 69(1):373-376

55. Ma YY, Zhou H, He HH, Jiao GT, Wei S (2019) A digital twin-based approach for quality control and optimization of complex product assembly. 2019 International Conference on Artificial Intelligence and Manufacturing A. http://dx.doi.org/10.1109/AIAM48774.2019.00157

56. Zhuang CB, Gong JC, Liu JH (2020) Digital twin-based assembly data management and process traceability for complex products. Journal of Manufacturing Systems

57. Zhuang CB, Liu JH, Xiong H (2018) Digital twin-based smart production management and control framework for the complex product assembly shop-floor. Int J Adv Manuf Technol 96(1-4):11491163

58. Zhuang CB, Liu JH, Xiong H, Weng G, Tang CT (2017) Assembly shop floor dynamic and real-time visual monitoring system for complex product. Comput Integr Manuf Syst 23(6):1264-1276

59. Weng G, Liu JH, He YX, Zhuang CB (2017) Quality data collection and management technology of aerospace complex product assembly process. 2017 5th International Conference on ComputerAided Design, Manufacturing, Modeling and Simulation. https://doi.org/10.1063/1.4981585

60. Meng SH, Tang SL, Zhu YH, Chen CY (2019) Digital twin-driven control method for robotic automatic assembly system. 2nd International Conference on Frontiers of Materials Synthesis and Processing. https://doi.org/10.1088/1757-899X/493/1/012128

61. Wu Y, Yao LY, Xiong H, Zhuang CB, Zhao HR, Liu JH (2019) Quality control method of complex product assembly process based on digital twin technology. Comput Integr Manuf Syst 25(6):15681575

62. Wang K, Liu DX, Liu ZY, Wang QD, Tan JR (2021) An assembly precision analysis method based on a general part digital twin model. Robotics and Computer-Integrated Manufacturing. https://doi.org/10.1016/j.rcim.2020.102089

63. Li L, Liu D, Liu JF, Zhou HG, Zhou JS (2020) Quality prediction and control of assembly and welding process for ship group product based on digital twin. Scanning2020: 1-13. https://doi.org/10.1155/2020/3758730 
64. Hu CM, Gao W, Xu CH, Ben KC (2020) Study on the application of digital twin technology in complex electronic equipment. Proceedings of the Seventh Asia International Symposium on Mechatronics. https://doi.org/10.1007/978-981-32-9441-7_14

65. Hao B, Wang MY, Fu SL, Xu DP, Wang JX (2020) Quality control mode of intelligent assembly workshop based on digital twin. Journal of Physics: Conference Series. https://doi.org/10.1088/1742-6596/1605/1/012036

66. Liu LY, Du HX, Wang HF, Liu TY (2019) Construction and application of digital twin system for production process in workshop. Comput Integr Manuf Syst 25(6):1536-1545

67. Wan F, Liu JH, Ning RX, Li ZJ (2012) Data collection and management method for discrete assembly process of complex product. Comput Integr Manuf Syst 18(8):1819-1828

68. Khan MN, Anwar S (2019) Time-domain data fusion using weighted evidence and dempster-shafer combination rule: application in object classification. Sensors. https://doi.org/10.3390/s19235187

69. Lv PL, Chen GS (2015) Fusion method of improved PSO \& D-S theory and application in intelligent diagnosis. Comput Integr Manuf Syst 21(8):2116-2123

70. Xu HH, Deng Y (2019) Dependent evidence combination based on decision-making trial and evaluation laboratory method. International Journal of Intelligent Systems 34(7):1555-1571

71. Sarikaya M, Gullu A (2015) Multi-response optimization of minimum quantity lubrication parameters using Taguchi-based grey relational analysis in turning of difficult-to-cut alloy Haynes 25 . J Clean Prod 91:347-357. http://dx.doi.org/10.1016/j.jclepro.2014.12.020

72. Wan LQ, Chen HZ, Ouyang LH, Zhang D, Wu JJ (2018) Multi-quality characteristics robust optimization design of complex equipment compliant mechanism based on hybrid Gray-FuzzyTaguchi method. Comput Integr Manuf Syst 24(6):1427-1437

73. Li H, Ye WH, Man ZG (2018) Markov localization algorithm for AGV based on Gaussian kernel function in feature map. Comput Integr Manuf Syst 24(5):1081-1088

\section{Figures}




\section{Intelligent Assembly Technology Based on Digital Twin}

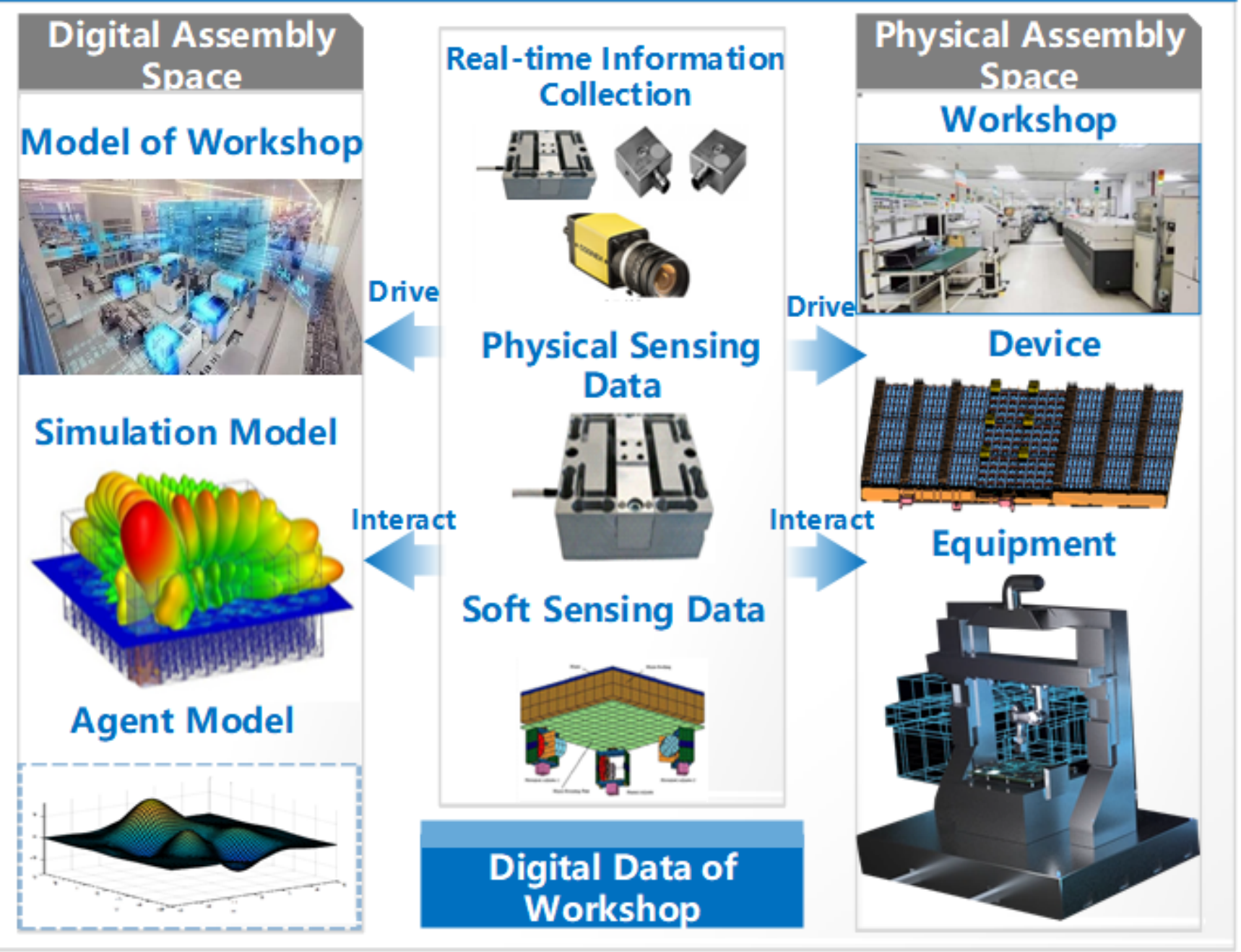

Figure 1

Digital twin-driven intelligent assembly technology 


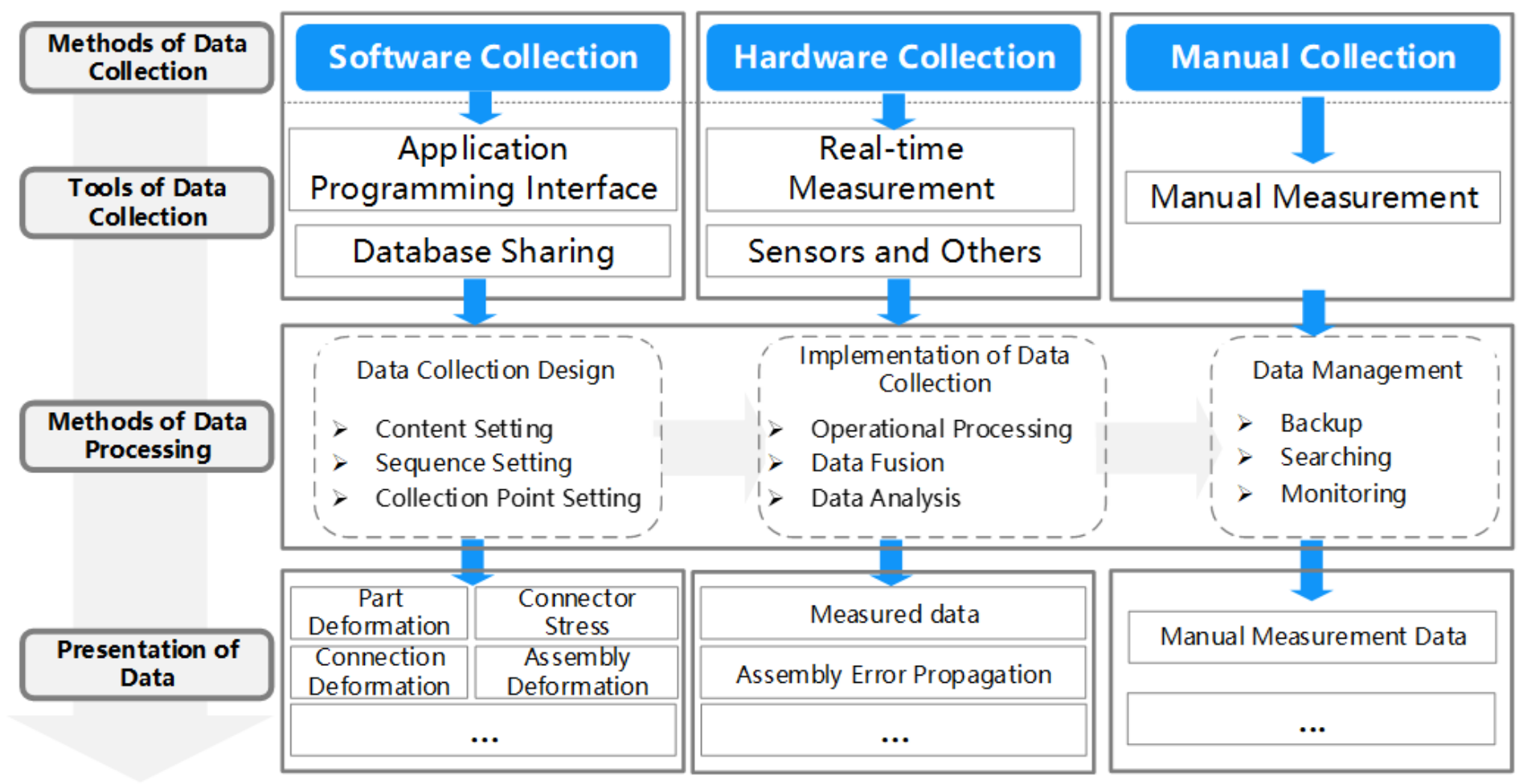

Figure 2

Flow of quality data collecting process in the assembly process of complex product 